\title{
Hypoxia-inducible factors and innate immunity in liver cancer
}

\author{
Vincent Wai-Hin Yuen' and Carmen Chak-Lui Wong ${ }^{1,2}$ \\ 'Department of Pathology and ${ }^{2}$ State Key Laboratory of Liver Research, University of Hong Kong, Hong Kong, China.
}

\begin{abstract}
The liver has strong innate immunity to counteract pathogens from the gastrointestinal tract. During the development of liver cancer, which is typically driven by chronic inflammation, the composition and biological roles of the innate immune cells are extensively altered. Hypoxia is a common finding in all stages of liver cancer development. Hypoxia drives the stabilization of hypoxia-inducible factors (HIFs), which act as central regulators to dampen the innate immunity of liver cancer. HIF signaling in innate immune cells and liver cancer cells together favors the recruitment and maintenance of pro-tumorigenic immune cells and the inhibition of anti-tumorigenic immune cells, promoting immune evasion. HIFs represent attractive therapeutic targets to inhibit the formation of an immunosuppressive microenvironment and growth of liver cancer.
\end{abstract}

\section{Introduction}

Liver cancer is a deadly malignancy with very limited treatment options. The liver contains a high number of residential immune cells, particularly innate immune cells, which enable the rapid clearance of harmful substances and pathogens from the gastrointestinal system. Liver cancer is an inflammation-driven disease preceded by hepatitis virus infection and steatosis. Immune cells are therefore abundant in the microenvironment of liver cancer. Many innate immune cells such as macrophages are immunosuppressive in liver cancer, and they reduce the treatment efficacy of immune checkpoint blockade. The level of hypoxia escalates with hepatocarcinogenesis. Hypoxia is a key feature of liver cancer and has an intimate link with innate immunity. In this Review, we will discuss how hypoxia-inducible factors (HIFs) control the biological roles of different innate immune cells and cancer cells to subvert the innate immune response, creating an immunosuppressive microenvironment in liver cancer. The implications of hypoxia in innate immune responses in normal and other pathological conditions are summarized in other excellent reviews $(1,2)$. We will conclude by discussing the potential of targeting HIFs and the immunosuppressive innate immune cells for treatment of hepatocellular carcinoma (HCC).

\section{Liver cancer}

Liver cancer ranks the fifth most common and the third most fatal cancer worldwide (3). The incidence of liver cancer and related deaths has risen over the past 20 years in the United States (4). HCC, the malignancy derived from hepatocytes that accounts for $75 \%-85 \%$ of primary liver cancers, will be the focus of this Review.

HCC's high mortality rate is caused by its late symptom presentation, high resistance to conventional chemo- and targeted

Conflict of interest: The authors have declared that no conflict of interest exists. Copyright: ( 2020 , American Society for Clinical Investigation.

Reference information: J Clin Invest. 2020;130(10):5052-5062.

https://doi.org/10.1172/JCl137553. therapies, and frequent recurrence. Currently, the tyrosine kinase inhibitors (TKIs) sorafenib, lenvatinib, and cabozantinib are the three FDA-approved first-line targeted drugs for advanced HCC, with a modest survival benefit (5-7). Nivolumab (an immune checkpoint inhibitor targeting PD-1) was recently approved by the FDA as a second-line treatment for sorafenib-resistant HCC. A clinical trial demonstrated that nivolumab has a response rate of only $20 \%$ in HCC patients (8).

Hepatocarcinogenesis develops in a stepwise manner: from chronic liver inflammation caused by viral infections or steatosis, to liver damage, to fibrosis and cirrhosis, and finally to HCC. HBV infection remains the major cause of HCC globally, while HCV infection and nonalcoholic steatohepatitis (NASH) associated with high-fat diet represent the major causes of HCC in Western countries (4). Toxic liver damage caused by alcohol abuse also contributes to HCC (4).

\section{Liver, liver cancer, and innate immunity}

Liver has a double circulation system, receiving $20 \%$ and $80 \%$ of blood supply from the hepatic artery and the portal vein, respectively. The hepatic artery delivers oxygenated blood to the liver. The portal vein delivers oxygen-depleted blood containing pathogens, bacterial products, food antigens, and environmental toxins from the gastrointestinal tract to the liver. Pathogens and toxic agents are cleared rapidly by the innate immune cells in the liver. The liver is enriched with innate immune cells including Kupffer cells (liver macrophages), natural killer (NK) cells, natural killer T (NKT) cells, and $\gamma \delta$ T cells (Figure 1 and refs. 9,10). HCC development is often preceded by chronic liver inflammation that is accompanied by increases in infiltrating innate immune cells (11). The most commonly found innate immune cells in HCC include myeloid-derived suppressor cells (MDSCs), macrophages, neutrophils, and NK cells (Figure 1 and ref. 11).

Myeloid cells are integral components of the innate immune system. MDSCs are a heterogeneous population of myeloid progenitors and immature myeloid cells and are generally classified 

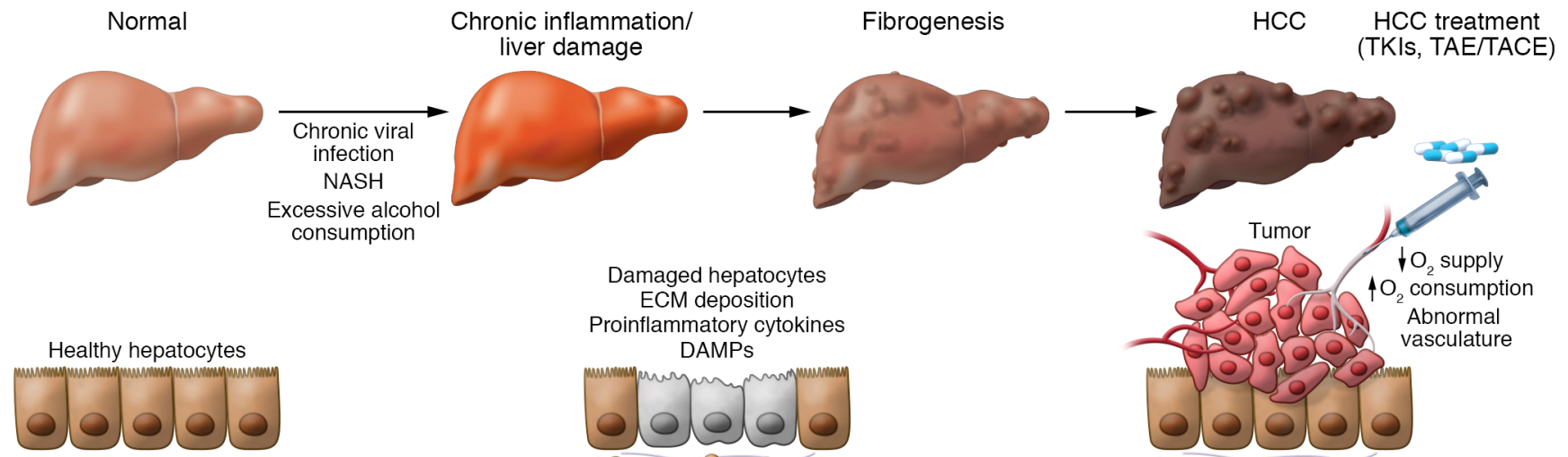

Damaged hepatocytes ECM deposition Proinflammatory cytokines DAMPs

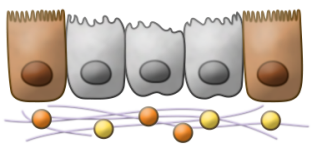

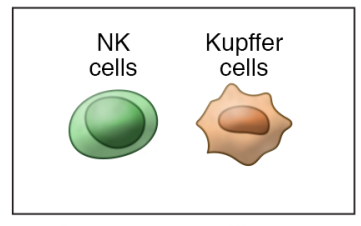

Immunosurveillance

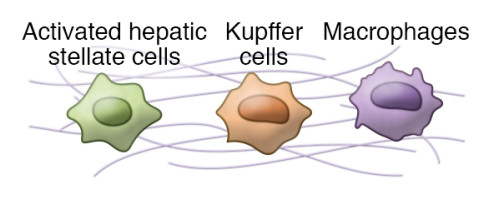

Profibrotic

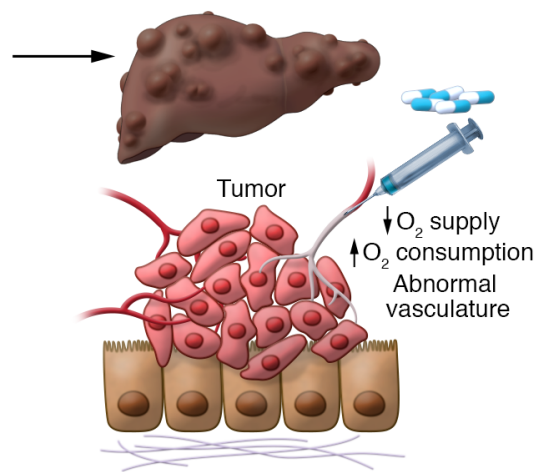

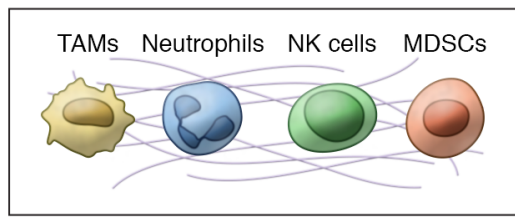

Immunosuppressive

Figure 1. Hypoxia, innate immunity, and the development of HCC. Hepatocarcinogenesis is a stepwise process. Chronic hepatitis virus infection and fatinduced steatosis (NASH), the major etiological factors of HCC, drive liver inflammation and tissue damage. Excessive alcohol consumption also induces liver damage. Damage in the liver disrupts the hepatic vasculature and perturbs proper blood flow and $\mathrm{O}_{2}$ supply, creating a hypoxic microenvironment. Hypoxic Kupffer cells, newly recruited macrophages, and hepatocytes activate hepatic stellate cells (HSCs) in the liver, which robustly deposit collagen, leading to fibrosis and then cirrhosis, which further intensifies hypoxia. When HCC is developed, hypoxia is even more severe. The growth of HCC outpaces the growth of blood vessels. Moreover, HCC cells consume all available $\mathrm{O}_{2}$. Different current HCC treatments, such as TAE/TACE and TKIs, further induce hypoxia. Hypoxia affects the activities of different innate immune cells, such as NK cells. Hypoxia also induces the infiltration and accumulation of many different types of immunosuppressive innate immune cells, including TAMs, MDSCs, and neutrophils, in the microenvironment of HCC. The $\mathrm{O}_{2}$ level decreases along with HCC development, driving the formation of an immunosuppressive microenvironment.

as granulocytic or polymorphonuclear MDSCs (PMN-MDSCs) and monocytic MDSCs (M-MDSCs) (12). Myeloid progenitors normally differentiate into mature macrophages, dendritic cells, and neutrophils. However, MDSCs remain undifferentiated, rapidly expand in cancer, and are highly immunosuppressive (13). MDSCs generate high ROS levels to suppress the $\mathrm{T}$ cell receptor (TCR) signaling pathway (13). MDSCs also express arginase 1 (ARG1), inducible NO synthase (iNOS), TGF- $\beta$, IL-10, COX-2, and indoleamine 2,3-dioxygenase (IDO) to suppress a variety of immune cells, including macrophages, dendritic cells, $\mathrm{T}$ cells, and NK cells (13).

Macrophages phagocytose pathogens. Macrophages found in tumors are collectively called tumor-associated macrophages (TAMs). In cancer, TAMs are thought to derive from circulating monocytes that originate from bone marrow-derived myeloid progenitors and are recruited to the tumor by the inflammatory factors secreted by cancer cells. At the tumor sites, tumor-secretory factors drive the differentiation of the recruited monocytes and MDSCs into TAMs. Kupffer cells also form a major population of TAMs in HCC. In cancer, macrophages are generally classified as M1 or M2 macrophages (14). M1 macrophages respond to damage-associated and pathogen-associated molecular patterns (DAMPs and PAMPs) and IFN- $\gamma$ and produce proinflammatory cytokines such as IL-12 (15). These proinflammatory macrophages are responsible for an antitumor response (14). M2 macrophages do not express MHC class II and have no antigen-presenting ability (15). They produce antiinflammatory cytokines including IL-4, IL-10, and IL-13 (15) and generally support tumor angiogenesis and cancer progression (15).

Neutrophils differentiate from PMN-MDSCs and are shortlived. Macrophages draw neutrophils to the site of infection through chemokines, where they are activated by binding to DAMPS/PAMPs. Activated neutrophils phagocytose foreign particles and sequester them into phagosomes, triggering the release of granules. In the cancer context, tumor-associated neutrophils (TANs) are classified as N1 (proinflammatory) and N2 neutrophils (antiinflammatory), exhibiting anti-tumorigenic and pro-tumorigenic roles, respectively (16).

NK cells belong to the innate lymphoid cell family and are nonphagocytic with rich cytoplasmic granules containing cytotoxic granzymes and perforin (17). NK cells rapidly induce cytolytic killing of cancer cells indirectly via degranulation and directly via death receptor cell signaling (TRAIL and Fas ligand) (17).

In general, cancer cells participate in the recruitment and maintenance of immunosuppressive cells and polarization of TAMs from M1 to M2. Hypoxia and HIFs play an important 


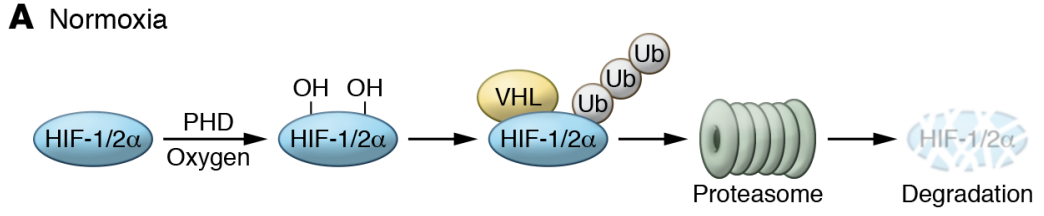

B Hypoxia

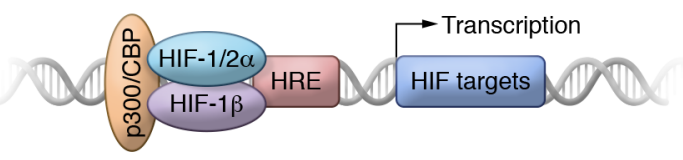

C

TAMs:

Suppression of $T$ cell cytotoxicity

Promotion of angiogenesis (VEGF, TIE2)

ECM remodeling (MMP-9)

Neutrophils:

Prevention of apoptosis

Recruitment of immunosuppressive cells, e.g., TAM, Treg

MDSCs:

ARG1, iNOS expression

Differentiation into TAM

Upregulation of PD-L1

NK cells:

Repression of NK activation receptors

role in accentuating the immunosuppressive characteristics of innate immune cells in the microenvironment of HCC.

\section{Establishment of hypoxic niche in liver cancer}

The $\mathrm{O}_{2}$ tensions in the periportal region and perivenous regions of normal liver are about $60-65 \mathrm{mmHg}$ and $30-35 \mathrm{mmHg}$, respectively (18). $\mathrm{O}_{2}$ tension drops to $6 \mathrm{mmHg}$ in liver cancer tissues (19). Hypoxia starts to develop in inflamed liver tissues as the result of viral infection or steatosis. The general mechanisms by which inflammation drives hypoxia are summarized in an excellent review (1). Hypoxia further accumulates at the time of liver injury and intensifies during fibrosis, cirrhosis, and HCC progression (Figure 1). Liver damage disrupts the hepatic vasculature, affecting blood flow and $\mathrm{O}_{2}$ delivery into areas of injured tissues (20). Cirrhosis further obstructs blood flow into the liver. In later stages of hepatocarcinogenesis, a number of causes further intensify hypoxia: $\mathrm{HCC}$ cells consume and deplete $\mathrm{O}_{2}$ quickly, but HCC is not supported by functional vasculature. HCC palliative therapy, such as transarterial embolization/chemoembolization (TAE/TACE), which aims to obstruct the blood supply to restrict tumor growth, inadvertently induces hypoxia (21). TKIs block multiple kinases responsible for angiogenesis, thereby further inducing intratumoral hypoxia (22).

\section{Mechanisms of HIF stabilization}

The major molecular mechanisms induced by hypoxia are mediated through HIFs, heterodimers composed of the $\mathrm{O}_{2}$-sensitive HIF- $1 / 2 \alpha$ subunit and the constitutively expressed HIF- $1 \beta$ subunit (refs. 23, 24, and Figure 2A). In the presence of $\mathrm{O}_{2}, \mathrm{HIF}-1 / 2 \alpha$ is hydroxylated at specific proline residues by prolyl hydroxylases (PHDs) (25). The von Hippel-Lindau (VHL) ubiquitin ligase complex recognizes the prolyl-hydroxylated HIF-1/2 $\alpha$ and con-
Figure 2. Roles of the hypoxia/HIF signaling pathway in innate immune cells in HCC. (A) In the presence of $\mathrm{O}_{2}$, the HIF- $1 / 2 \alpha$ subunit is hydroxylated by the PHD enzymes at two specific proline residues, enabling the binding of $\mathrm{VHL}$. VHL targets the hydroxylated HIF-1/2 $\alpha$ subunit for ubiquitin-mediated proteasomal degradation. (B) In the absence of $\mathrm{O}_{2}$, the HIF-1/2 $\alpha$ subunit is stabilized and dimerizes with HIF-1 $\beta$, together with cotranscriptional factors p300 and $C B P$, to drive the transcription of genes encompassing hypoxia-responsive elements (HREs). (C) The hypoxia/ HIF signaling pathway in the innate immune cells directly affects their properties in HCC. jugates HIF-1/2 $\alpha$ with ubiquitin, directing HIF- $1 / 2 \alpha$ for proteasomal degradation (26). In the absence of $\mathrm{O}_{2}, \mathrm{HIF}-1 / 2 \alpha$ is stabilized and dimerizes with HIF-1 $\beta$, together with the coactivator p300/CBP, to drive transcription of genes encompassing hypoxia-responsive elements, which are the conserved core sequence 5'-RCGTG-3', in different cell types that experience hypoxia (27).

\section{Hypoxia and HIFs in early stages of hepatocarcinogenesis}

HIF protein induction can be detected in early diseased liver tissues and contributes to early steps of hepatocarcinogenesis (20). In the fibrotic liver, hypoxic Kupffer cells and newly recruited macrophages function as M1 macrophages (Figure 1). These proinflammatory cells and hepatocytes generate a high level of secretory growth factors, including PDGF- $\beta$, in a HIF-dependent manner (28-30). PDGF- $\beta$ promotes proliferation of hepatic stellate cells (HSCs) in the liver (31). HSCs differentiate into myofibroblasts, which actively produce collagen, causing fibrosis and cirrhosis (32). Hypoxia also induces VEGF secretion in Kupffer cells and HSCs $(28,33)$. VEGF activates HSCs, promotes fibrogenesis, and recruits monocytes $(32,34,35)$. Apart from effects on PDGF- $\beta$ and VEGF, HIFs drive the expression of a list of proinflammatory cytokines and fibrogenic genes. Mice with hepatocyte-specific $V h l$ knockout demonstrated increased HIF-1/2 $\alpha$ expression and an induction of fibrotic genes, including collagen synthesizing and modification enzymes (Plod2, Tgm2) and $\alpha$-SMA (a marker for activated myofibroblasts) (36). Deleting Hifla in HSCs reduces collagen synthesis and impedes macrophage-mediated clearance of necrotic cells in damaged liver (37). All these studies unequivocally demonstrated that HIF signaling in all liver cell types is critical in driving early hepatocarcinogenesis. 
Table 1. HIFs' roles in innate immune cells and their crosstalk in HCC and other disease models

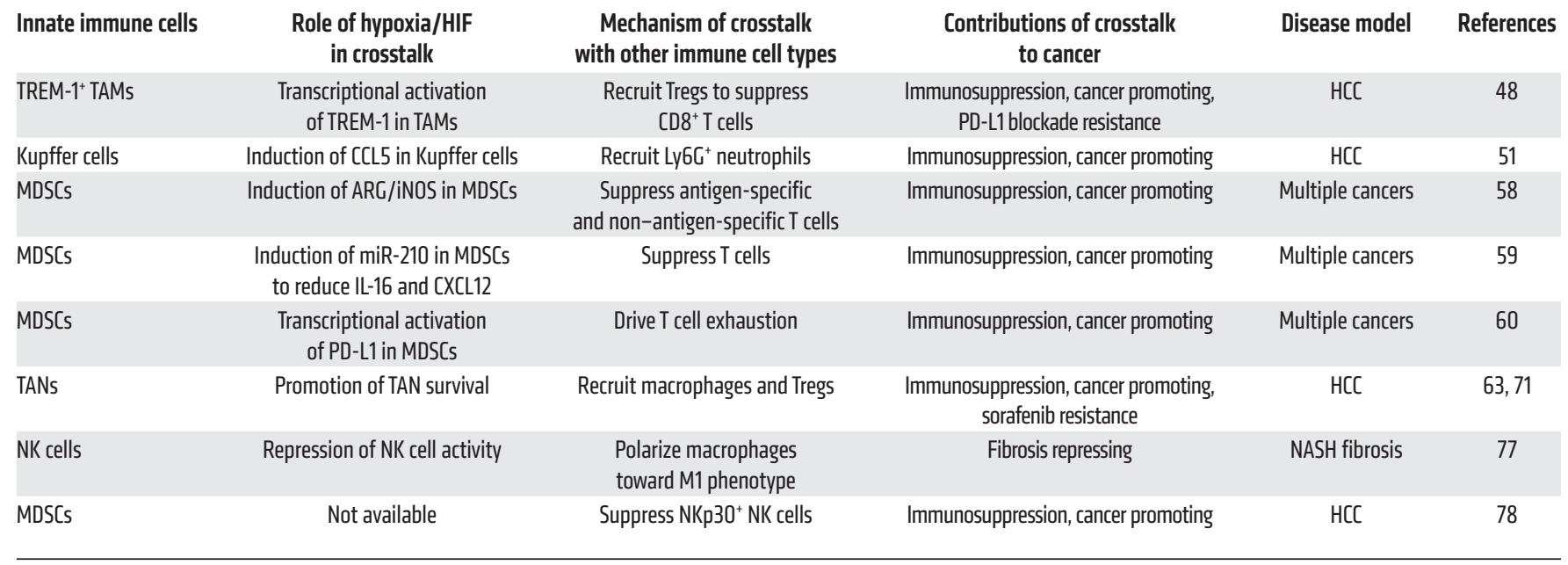

\section{Hypoxia and HIFs in later stages of hepatocarcinogenesis}

HIF- $1 / 2 \alpha$ protein is highly expressed in human HCC tissues (38-40), and expression correlates with poor clinical outcome in HCC patients $(41,42)$. A hypoxic score based on seven hypoxia-related genes displayed strong prognostic values in HCC (43). Interestingly, a recent single-cell RNA sequencing study demonstrated that liver cancers infiltrated with higher amounts of nonmalignant cells, including TAMs, have stronger hypoxic signaling (44). Another single-cell RNA sequencing study on tumor-infiltrated immune cells confirmed that HCC contains a variety of innate immune cells, including conventional NK cells, liver-resident NK cells, MDSCs, and TAMs (45). Notably, it was found that TAMs coexpress M1 and M2 gene signatures, consolidating the recent perception on the complicated biology of TAMs beyond the M1 and M2 classifications (45). TAMs may exist in a continuum of functional states (14), and coexistence of proinflammatory and antiinflammatory macrophages is possible in human HCC.

\section{HIFs' roles in innate immune cells in HCC}

HIFs in TAMs. HIF- $1 \alpha$ and HIF- $2 \alpha$ have distinct roles in myeloid cells. HIF-1 $\alpha$ supports glycolysis and ATP production in myeloid cells, enabling them to infiltrate and adhere at inflammatory tissues (46). HIF-2 $\alpha$ drives the expression of receptors such as CXCR4, M-CSFR, and fibronectin 1 (FN1) in myeloid cells, enabling them to migrate to and infiltrate inflammatory and cancer tissues (47). In a diethylnitrosamine-induced mouse HCC model, deletion of HIF- $2 \alpha$ in myeloid cells suppressed TAM infiltration into HCC (47). HIF- $1 \alpha$ also transcriptionally activates the receptor TREM-1 in TAMs (48). TREM-1+ TAMs recruit immunosuppressive Tregs to HCC in a hypoxia-dependent manner, leading to reduced infiltration of $\mathrm{CD}^{+} \mathrm{T}$ cells and poor survival in human HCC patients (Figure 2B and ref. 48). NF- $\kappa \mathrm{B}$ was shown to induce Hifla mRNA in macrophages (49). HIF-1 $\alpha$ protein was reported to be degraded by hypoxia-associated factor (HAF; encoded by SART1), an E3 ubiquitin ligase, in an oxygen-independent manner (50). The
Kupffer cells in Sart1 heterozygous KO mice constitutively express HIF-1 $\alpha$, which induces RANTES/CCL5 to recruit Ly6G ${ }^{+}$neutrophils into the liver, driving HCC (51).

In addition to the primordial functions of TAMs in T cell suppression and immune evasion, TAMs also promote angiogenesis. Hypoxia induces an array of angiogenic factors (VEGF, PDGF- $\beta$, FGF, angiopoietins) in primary human macrophages to promote angiogenesis (ref. 52 and Figure 2B). The induction of VEGF in macrophages was directly shown to be mediated through HIF-1 $\alpha$ (53). Apart from directly secreting angiogenic factors, hypoxic TAMs release MMP-9, which cleaves the extracellular matrix to liberate the sequestered VEGF (ref. 54 and Figure 2B). Moreover, hypoxia induces TIE2, a monocyte receptor. TIE2-expressing monocytes (TEMs) represent a strong proangiogenic myeloid subset and are attracted by endothelial cell-derived angiopoietin-2 in hypoxic tumors (55). Interestingly, TAM/TEM infiltration is frequently found in HCC tissues and is positively correlated with microvessel density, with prognostic value in $\operatorname{HCC}(56,57)$.

HIFs in MDSCs. An interesting study comparing the difference of splenic MDSCs and tumor-associated MDSCs in tumor-bearing mice has highlighted the roles of hypoxia in MDSCs (58). The concentration of $\mathrm{O}_{2}$ in the spleen is higher than that in tumors. Splenic MDSCs in tumor-bearing mice suppress antigen-specific $\mathrm{T}$ cells through ROS induction (58). Hypoxia in the tumor activates HIF- $1 \alpha$ in tumor-associated MDSCs, thereby driving ARG1 and iNOS expressions to suppress both antigen-specific and non-antigen-specific T cells (ref. 58 and Figure 2B). Along these lines, an interesting study showed that a well-known HIF-1 $\alpha-$ induced microRNA, miR-210, is highly expressed in tumorassociated MDSCs as compared with splenic MDSCs (59). MiR210 in tumor-associated MDSCs increases ARG1 and reduces IL-16 and CXCL12 to suppress T cells (59). HIF-1 $\alpha$ drives the differentiation of tumor-associated MDSCs into TAMs to further promote tumorigenesis (ref. 58 and Figure 2B). It is of great interest that HIF- $1 \alpha$ transcriptionally activates PD-L1 in MDSCs and other myeloid cells (ref. 60 and Figure 2B). PD-L1 is a well-known immune checkpoint surface protein that drives $\mathrm{T}$ cell exhaustion 

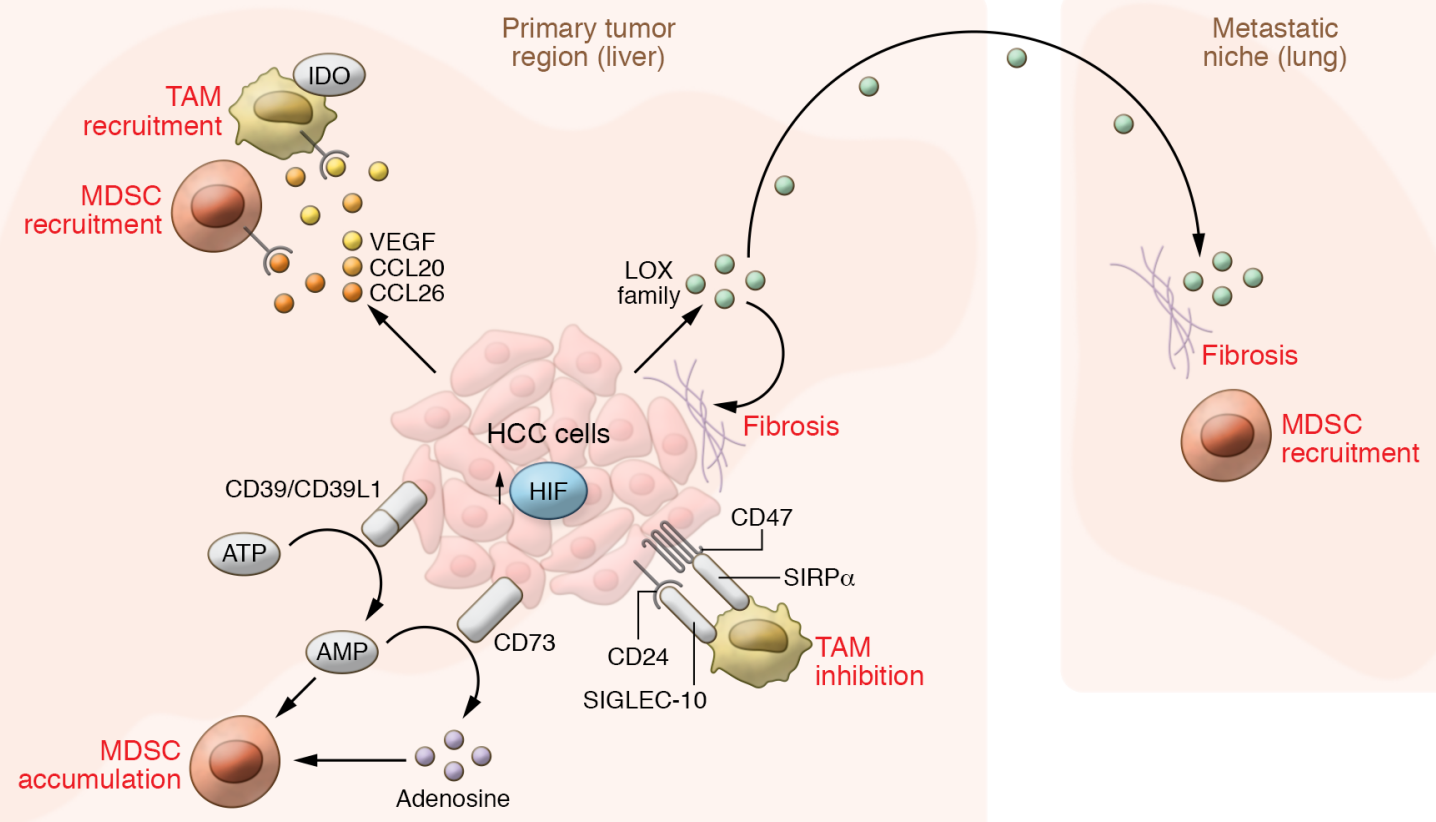

Figure 3. Roles of the hypoxia/HIF signaling pathway in HCC cells that affect innate immune cells in the tumor microenvironment. HIFs activate the transcription and secretion of the chemokines CCL20, VECF, and CCL26 in HCC cells. These chemoattractants recruit immunosuppressive TAMs and MDSCs to HCC. HIFs activate the transcription of the don't-eat-me signal surface markers (CD47, CD24) in cancer cells including HCC cells. CD47 and CD24 are well-characterized liver cancer stem cell markers. CD47 and CD24 prevent cancer cells from being phagocytosed by the macrophages. HIFs activate the transcription of members of the purinergic signaling pathway (CD39, CD39L1, CD73) in HCC cells to create an adenosine- and AMP-rich microenvironment that favors the accumulation of MDSCs. HIFs activate the transcription of members of the LOX family in HCC cells. The LOX family cross-links collagen in the primary cancer and metastatic niches. At the primary liver cancer niche, the LOX family increases tissue stiffening and promotes local invasion of cancer cells. At the metastatic niche in the lung, cross-linking of collagen mediated by the LOX family helps to recruit MDSCs, creating a favorable niche for HCC cell colonization.

through binding to PD-1, an inhibitory receptor on T cells (61). PD-L1 expression on monocytes in tumors is associated with poor survival in HCC patients, and blockade of PD-L1 reinstates T cell immunity against HCC (62).

HIFs in neutrophils. Hypoxia promotes survival and prevents apoptosis of neutrophils through HIF-1-mediated transcription of NF- $\mathrm{kB}$ (ref. 63 and Figure 2B). Cancer cells secrete high levels of G-CSF and other chemokines such as CXCL1, CXCL2, CXCL5, and CXCL8 to recruit and activate neutrophils $(64,65)$. TGF- $\beta$ then polarizes TANs into immunosuppressive $\mathrm{N} 2$ phenotype with high ARG1, CCL2, and CCL5 expression (66). Depleting neutrophils activates $\mathrm{CD} 8^{+} \mathrm{T}$ cells and represses HCC growth $(66,67)$. Interestingly, TGF- $\beta$ and HIF- 1 could be reciprocally induced (68, 69) and activate the same genes in HCC (70). Thus, hypoxia/HIF1 might also contribute to N2 polarization of neutrophils. HCC cells activate the PI3K/AKT and p38/MAPK pathways in TANs to induce their expression of CCL2 and CCL17 (71). TANs, through CCL2 and CCL17, recruit macrophages and Tregs to HCC through CCR2 and CCR4 receptors (71). Apart from attracting immunosuppressive cells, TANs also promote angiogenesis in tumors. Interestingly, increased TAN infiltration was found in tumors from HCC patients treated with sorafenib (71). Sorafenib treatment, through inducing hypoxia and HIF-1 stabilization, activates the NF-kB pathway in HCC cells to drive CXCL5 secretion, which further attracts TANs to HCC (71). Depletion of TANs sensitizes HCC to sorafenib treatment in mice (71). In a large cohort of 452
HCC patients, immunohistochemical study confirmed that the number of TANs significantly correlated with the number of M2 macrophages and Tregs as well as poor prognosis (71), indicating the immunosuppressive roles of TANs in HCC.

Hypoxia, HIFs, and NK cells. NK activity is balanced between signals from NK activation receptors and NK inhibitory receptors. NK activation receptors bind to ligands expressed by infected and cancer cells. NK inhibitory receptors bind to the classical MHC class I molecule, which is only expressed by host cells to prevent them from being mistakenly attacked by NK cells. The NK activation receptors, including NKp46, NKp44, NKp3O, and NKG2D, are repressed by hypoxia (ref. 72 and Figure 2B). Meanwhile, ligands that bind to NK activation receptors are repressed in cancer, thereby preventing NK cell-mediated cancer cell killing. In HCC, expression of NKG2D ligand was reduced in more aggressive HCC (73). Nonclassical MHC class Ib molecules (HLA-E, HLA-F, HLA-G), which are often upregulated in cancer, can bind to NK inhibitory receptors to inhibit NK cells. HLA-G was shown to be a HIF-1 transcriptional target in cancer (74). In HCC, HLA-G was expressed in over half of human HCC tissues $(75,76)$. It is therefore rational to speculate that NK cell activity is partially limited in the tumor microenvironment of HCC. NK cells also crosstalk with myeloid cells during different stages of hepatocarcinogenesis and polarize M2 to M1 macrophages to suppress NASH development to fibrosis (77). Interestingly, MDSCs are able to suppress NK cells in HCC (78). 
Table 2. Roles of HIFs in HCC cells

\begin{tabular}{|c|c|c|c|c|c|}
\hline $\begin{array}{l}\text { HIF transcriptional target } \\
\text { in cancer cells }\end{array}$ & $\begin{array}{l}\text { Overexpression } \\
\text { in HCC }\end{array}$ & $\begin{array}{l}\text { Direct effect on innate immune cells } \\
\text { in the tumor microenvironment }\end{array}$ & $\begin{array}{l}\text { Mechanisms of crosstalk } \\
\text { with other immune cell types }\end{array}$ & Role in cancer & References \\
\hline SEMA3A & Yes & Attracts NRP1+ ${ }^{+}$TAMs to tumors & $\begin{array}{l}\text { Suppresses proliferation } \\
\text { and recruitment of T cells }\end{array}$ & Cancer promoting & $82-84$ \\
\hline VEGF & Yes & $\begin{array}{l}\text { Recruits VECFR1 } 1^{+} \text {monocytes } \\
\text { and macrophages to tumors }\end{array}$ & Not available & Cancer promoting & $85-88$ \\
\hline LOXL2 & Yes & Recruits MDSCs to metastatic niche & Not available & Metastasis promoting & 70,92 \\
\hline CD47 & Yes & Prevents phagocytosis by macrophages & Not available & Cancer promoting & $95,96,99$ \\
\hline CD24 & Yes & Prevents phagocytosis by macrophages & Not available & Cancer promoting & $\begin{array}{c}94,97,98 \\
100\end{array}$ \\
\hline CD39L1 & Yes & $\begin{array}{l}\text { Enriches MDSCs by preventing their } \\
\text { differentiation to macrophages and } \\
\text { dendritic cells }\end{array}$ & Suppresses T cells & $\begin{array}{l}\text { Cancer promoting, } \\
\text { immune checkpoint blockade } \\
\text { resistance }\end{array}$ & 118 \\
\hline
\end{tabular}

These data together suggest that HIF signaling in different immunosuppressive innate immune cells generally contributes to poor outcome in HCC (Table 1). Most studies mentioned above focus on the roles of HIF in cytokine induction in different innate immune cells. HIF-1 is a central regulator of metabolism in all cell types by diverting metabolites into glycolysis. It is likely that all cell types in the hypoxic tumor niche are forced to take the glycolytic route. The current literature has not addressed how HIF1-mediated metabolic switching affects the biology of different tumor-infiltrated immune cells in HCC, nor, importantly, whether simultaneous activation of HIF- 1 in both immune and cancer cells leads to nutrient competition between different immune cells and cancer cells in the hypoxic HCC microenvironment. Currently, innate immune cells are characterized by limited markers using flow cytometry. Single-cell RNA sequencing will bring new knowledge about the heterogeneity of each innate immune cell type. Integration of this information with hypoxia gene signature will bring new insight into the roles of hypoxia and HIFs in the biology of different immune cells.

\section{Roles of HIFs in HCC cells}

HIFs in cancer cells regulate a repertoire of genes responsible for maintaining an immunosuppressive microenvironment in the tumor. These HIF-regulated genes encode for chemoattractants, members in the purinergic signaling pathway, and don't-eat-me signaling molecules that allow cancer cells to evade immune surveillance (Figure 3 and Table 2). Notably, hypoxia also induces cancer cell death, which attracts macrophages.

HIFs induce chemoattractants and lysyl oxidase (LOX) enzymes. HIFs in cancer cells transcriptionally activate CCL20, SEMA3A, and VEGF to enrich TAMs and CCL26 to recruit MDSCs. Transcriptional regulation of HIFs on these genes has been demonstrated in multiple solid cancer types, including HCC. All of these genes are overexpressed in human HCC.

CCL2O is a chemokine that is transcriptionally activated by HIF-1 in human HCC cells (79). CCL2O induces the expression of the immunosuppressive enzyme IDO in macrophages through the STAT pathway in the HCC microenvironment (79). IDO metabolizes tryptophan (Trp) to kynurenine (Kyn) (80). Trp supports T cell proliferation, while Kyn binds to AHR transcription factor of $\mathrm{CD}^{+}$ $\mathrm{T}$ cells to suppress their differentiation to Th17 cells and favor the production of Tregs (81). CCL20-treated IDO+ macrophages enrich Tregs and suppress proliferation and IFN- $\gamma$ production of effector T cells in HCC (79). Interestingly, HCC patients with high CCL2O expression have shorter survival. CCL20 and IDO expressions are also correlated in portal vein tumor thrombus (79).

SEMA3A is a secretory factor that attracts neuropilin-1expressing (NRP1-expressing) macrophages. In a lung cancer model, hypoxia induced SEMA3A secretion to attract NRP1 ${ }^{+}$ TAMs to tumors (82). SEMA3A activates PlexinA1/PlexinA4 sig-

\section{Table 3. HIF inhibitors used in HCC}

HIF inhibitor

Digoxin

EZN-2968

HIF-1 $\alpha$ antisense oligonucleotide

PT-2385
Mechanism of action Suppresses translation of HIF-1 $\alpha$ Locked nucleic acid targeting HIF-1 $\alpha$ Antisense nucleic acid targeting HIF-1 $\alpha$ Inhibits HIF-1 $\alpha$ protein Suppresses heterodimerization of the HIF-2
Effect in HCC

Suppresses HCC growth and metastasis in mice

References

Partial response and stable disease in a small subset of HCC patients Improves the efficiency of TAE in HCC-bearing rats

Synergizes with sorafenib to suppress HCC growth in mice Synergizes with sorafenib to inhibit HCC growth in mice
$70,89,130$

135,136

21

137,138

139 
naling to stimulate VEGFR1 on TAMs, inducing motility toward hypoxic cancer regions (82). More interestingly, Casazza et al. further showed that after NRP1+ TAMs enter hypoxic zones, HIF- $2 \alpha$ activates IKK complex (via the NF- $\mathrm{BB}$ pathway) to drive feed-forward repression of NRP1 expression on TAMs, preventing these TAMs from exiting the hypoxic zone (82). Multiple studies have reported that SEMA3A is overexpressed in human $\operatorname{HCC}(83,84)$.

VEGF is a well-known transcriptional target of HIF that promotes tumor angiogenesis. VEGF also activates VEGFR1 (also known as FLT-1) on monocytes and macrophages to recruit them $(85,86)$. Hypoxia induces VEGF expression in human HCC cell lines by activating $V E G F$ gene transcription and increasing $V E G F$ mRNA stability. VEGF-expressing HCC cells are in close proximity to the hypoxic tumor region. Overexpression of VEGF is observed in HCC and is correlated with tumor vascularization and progression $(87,88)$.

CCL26 is chemotactic for eosinophils and monocytes $(89,90)$. Our group has reported that CCL26 is a transcriptional target of HIFs in HCC cells (89). Hypoxia-induced CCL26 in HCC cells attracts CX3CR1 ${ }^{+}$MDSCs to the tumor site (89). We showed that tumor-associated MDSCs promote HCC growth by enhancing angiogenesis. Blocking communication between HCC cells and MDSCs using HIF inhibitor or CX3CR1 neutralizing antibody prevents MDSC homing and suppresses HCC development in mice (89).

Apart from regulating chemokines, HIFs were shown to recruit MDSCs to the metastatic site through transcriptional activation of the lysyl oxidase (LOX) family in cancers $(91,92)$. LOX family members, including LOX and LOXL1-LOXL4, are secretory amine oxidases that posttranslationally modify and cross-link collagen. In HCC, our group found that hypoxia-induced LOXL2 is responsible for cross-linking collagen in the primary tumors (70). Overexpression of LOXL2 protein is found in tissues and sera of HCC patients (70). At the primary site, cross-linking of collagen promotes the local invasion of HCC cells by enhancing tumor stiffness, which activates the Rho kinase-mediated cytoskeletal remodeling of HCC cells (70). At the metastatic site, cross-linking of collagen facilitates the attachment of myeloid cells $(70,92)$. These myeloid cells are known to be rich in angiogenic factors that prime the metastatic tissues for cancer cell lodging and colonization (91). It will be interesting to comprehensively examine the immunosuppressive roles of this subset of myeloid cells in the metastatic niche. An elegant study showed that HBV infection triggers the expansion of PMN-MDSCs, which protect the liver from T cell-induced inflammation and liver damage (93). HCC might represent a unique cancer type with an exceptionally high number of MDSCs due to HBV infection. Previously, our group showed that MDSCs preferentially localize at hypoxic regions of human HCC (89). Hypoxia/HIFs might act as a second trigger to further recruit MDSCs through transcriptional induction of various chemoattractants in HCC cells. It will be interesting to evaluate the differences between MDSCs in hepatitis-associated HCC and steatosis-associated HCC.

HIFs induce don't-eat-me signals to prevent phagocytosis. Hypoxia induces the "don't-eat-me" signals CD47 and CD24 through HIF-1-mediated transcription in cancer cells to evade phagocytosis by macrophages $(94,95)$. CD47 expressed on cancer cells binds to SIRP $\alpha$ on macrophages. The cytoplasmic domain of SIRP $\alpha$ contains the immunoreceptor tyrosine-based inhibition motifs
(ITIMs), which relay inhibitory signals to suppress phagocytosis (96). CD24 expressed on cancer cells binds to SIGLEC-10, a receptor on macrophages that inhibits phagocytosis (97). Both CD47 and CD24 are liver cancer stem cell markers $(98,99)$. CD24+ liver cancer stem cell population is directly induced by hypoxia (100). Liver cancer stem cells are populations of liver cancer cells with self-renewal capabilities and high resistance toward chemo- and targeted therapies (101). These findings collectively suggest a new role of hypoxia-enriched liver cancer stem cells in evading surveillance from the innate immune system.

HIFs induce purinergic signaling to evade immune surveillance. ATP is released extracellularly by injured or dying cells. Extracellular ATP is generally proinflammatory and serves as a danger signal to direct phagocytic cells to the inflamed tissues and to alarm other immune cells. A pair of ectoenzymes, CD39/CD39L1 (ENTPD1/ ENTPD2) and CD73 (NT5E), are responsible for the conversion of extracellular ATP to adenosine (102). CD39/CD39L1 dephosphorylates extracellular ATP to ADP to AMP, while CD73 further dephosphorylates extracellular AMP to adenosine. Adenosine is immunosuppressive and accumulates in hypoxic tissues $(103,104)$. Extracellular adenosine is sensed by $G$ protein-coupled receptors (A2AR and A2BR) on immune cells. Through A2AR, adenosine inhibits the activity of M1 macrophages and NK cells, increases PD- 1 and CTLA-4 expression on T cells, and enriches Tregs (105-112). A2AR also inhibits secretion of proinflammatory cytokines such as IFN- $\gamma$ and IL-2 $(108,111)$. Through A2BR, adenosine promotes M2 macrophages and MDSCs, and prevents antigen presentation of dendritic cells (107, 113-115). CD39, CD39L1, and CD73, well-known cancer therapeutic targets $(105,116,117)$, are transcriptionally activated by HIFs in different cell types, including HCC (118-120). Hypoxia also induces A2BR in cancer cells and dendritic cells in a HIF-dependent manner $(115,121)$. Mechanistically, CD39, CD39L1, and CD73 drive extracellular adenosine accumulation and immunosuppression in different cancer models. Our group showed that HIF-1-induced CD39L1 in HCC cells suppresses the differentiation of M-MDSCs to dendritic cells through AMP, thereby maintaining MDSCs and limiting $\mathrm{T}$ cell activity in the hypoxic niche (118). We further showed that CD39/CD39L1 inhibitor synergizes with anti-PD-1 to inhibit HCC development in mice (118). Effects of ATP and adenosine are broad given the ubiquitous expression of their receptors in multiple cell types leading to paradoxical effects in the pathogenesis of diseases. In $\mathrm{Cd} 39-\mathrm{KO}$ mice, it was shown that ATP activates several key oncogenic pathways in the liver; therefore CD39 loss promotes the formation of carcinogen-induced HCC in mice (122). In Cd39l1-KO mice, it was shown that CD39L1 protects mice from liver fibrosis (123). Interestingly, two elegant studies demonstrated that CD39 on myeloid cells protects mice from sepsis-associated liver injury and biliary fibrosis in sclerosing cholangitis $(124,125)$. Cd39-KO and Cd73-KO mice were more susceptible to liver injury and acute liver failure (126). Taken together, these studies suggest that, in the cancer context, conversion of extracellular ATP to AMP or adenosine creates an immunosuppressive microenvironment. Ectoenzymes involved in this conversion and receptors responding to these immunosuppressive metabolites are all induced by hypoxia and regulated by HIFs; therefore, hypoxia and HIFs play a central role in purinergic signaling and contribute to immune evasion. 
Hypoxia-mediated necrotic debris mediates HCC and macrophage communication. Hypoxia often results in necrosis in cancer tissues. Macrophages are dead-cell scavengers that are recruited to necrotic regions to clear cellular debris. An interesting study has demonstrated that necrotic debris from severe hypoxic HCC cells initiates crosstalk between cancer cells and TAMs in hypoxic HCC (127). The researchers observed that CD206 ${ }^{+}$TAMs (M2) are preferentially located in necrotic regions in human HCC (127). The necrotic debris from hypoxic HCC cells polarizes macrophages by inducing M2 markers (CD163, ARG1, and MRC1) and repressing M1 markers (iNOS) (127). The necrotic debris from hypoxic HCC cells also induces IL-1 $\beta$ through TLR4/TRIF/NF- $\kappa B$ signaling in macrophages (127). Intriguingly, IL-1 $\beta$, through the NF- $\kappa \beta / C O X-2$ pathway, in turn induces HIF-1 $\alpha$ transcription in HCC cells, thereby activating the epithelial-mesenchymal transition of HCC cells (127).

Compared with other cancer types, the available literature regarding the roles of HIF signaling in immune evasion in HCC is scanty. This can be attributed to the lack of reliable transgenic mouse models that can form spontaneous HCC, preventing researchers from studying the immune microenvironment of HCC. Hydrodynamic tail-vein injection enables the delivery of the genome editing systems to the mouse liver to knock out tumor suppressor genes and overexpress oncogenes to induce HCC in immune-competent mice $(128,129)$. This technological advancement is expected to substantially expedite research in this area.

\section{HIF inhibitors as HCC treatment}

A number of drugs have been identified to suppress different steps contributing to HIF functions. These drugs mainly inhibit the transcription, translation, dimerization, DNA binding, and transcriptional activity of HIFs. We will focus on the HIF inhibitors that have been evaluated in liver cancer (Table 3). An elegant drug library screening study in a human HCC cell line identified digoxin as an effective HIF-1 inhibitor (130). Digoxin suppresses the translation of HIF- $1 \alpha$ and inhibits multiple HIF-1-mediated pro-tumorigenic events (130-134). We first demonstrated that digoxin is able to repress HIF-1/LOX family-mediated premetastatic niche formation, including collagen cross-linking, recruitment of CD11b ${ }^{+}$ myeloid cells, and cancer cell colonization in breast cancer (70). In HCC, our group showed that digoxin effectively inhibits tumor growth and suppresses the recruitment of MDSCs to the primary tumor site (70). Digoxin is currently being evaluated in clinical trials for breast cancer (ClinicalTrials.gov NCT01763931) and pancreatic cancer as a combined treatment with FOLFIRINOX (NCT04141995). While the effects of digoxin in human HCC remain to be investigated, a phase Ib clinical trial on EZN-2968 (135), a locked nucleic acid targeting HIF-1 $\alpha$, showed that two of eight HCC patients had partial response and stable disease (136).

A number of HIF inhibitors could improve the effects of existing HCC treatments (Table 3). A study showed that intraportal delivery of HIF-1 $\alpha$ antisense oligonucleotides via adeno-associated viral vector is able to improve the efficiency of TAE in HCC-bearing rats (21). The clinical outcomes of sorafenib, lenvatinib, and cabozantinib, like many antiangiogenic inhibitors, have been disappointing, as tumors often develop resistance. One of the important mechanisms that contribute to antiangiogenic inhibitor resistance is the undesirable induction of intratumoral hypoxia and stabilization of HIFs. As demonstrated in a mouse model, sorafenib induces HIF-1 $\alpha$ in HCC, leading to sorafenib resistance (137). A drug with antagonistic effects against HIF$1 \alpha$, EF24, was able to synergize with sorafenib to suppress HCC growth in mice $(137,138)$. A potent HIF-2 inhibitor, PT-2385, which specifically suppresses the heterodimerization of HIF-2, synergizes with sorafenib to inhibit HCC growth (139). Meanwhile, another study showed that sorafenib promotes the recruitment of myeloid cells expressing a high level of macrophage chemoattractants in a mouse HCC model (140). Cotreatment of sorafenib and TAM-depleting agents (zoledronic acid or clodrolip) synergistically inhibits HCC angiogenesis, growth, and metastasis (140). These studies independently show that inhibition of HIFs or inhibition of TAM synergizes with sorafenib in HCC. Whether the effects of HIF inhibition are mediated through TAM inhibition in the microenvironment of HCC merits further exploration. An additional consideration is that TAM-depleting agents simultaneously remove pro-tumorigenic and anti-tumorigenic TAMs. Therapeutic agents that specifically drive the polarization of TAMs to reinvigorate their phagocytic, antigen-presenting, and other antitumoral abilities are yet to be developed.

A recent phase III clinical trial (IMbrave 50) showed that combined anti-PD-L1 (atezolizumab) and anti-VEGF (bevacizumab/ Avastin) therapy as a first-line treatment remarkably extended overall and progression-free survival in advanced HCC patients (141). This is the first drug regimen to show improved survival benefits relative to sorafenib with statistical significance and will likely change the clinical treatment of HCC in the near future (141). Synergy of antiangiogenic treatment with immune checkpoint blockade was concurrently observed in a mouse HCC model (142), in which anti-VEGFR2 stimulates PD-1 and PD-L1 expression in vivo (142). Bevacizumab induces intratumoral hypoxia $(143,144)$. It is rational to speculate that antiangiogenic drugs like bevacizumab induce an immunosuppressive microenvironment through HIF-1 by enriching TAMs, MDSCs, and TANs and inducing PD-L1, which together limit T cell activity. Therefore, PD-1/PD-L1 blockade restores T cell activity and works synergistically with antiangiogenic drugs, explaining the strong synergy observed in atezolizumab and bevacizumab in HCC patients.

\section{Conclusion}

Hypoxia is a key microenvironmental factor in all stages of hepatocarcinogenesis. Hypoxia affects all cell types in the liver. In early stages of hepatocarcinogenesis, HIFs in Kupffer cells promote liver cirrhosis by activating HSCs to generate profibrogenic factors. In later stages of hepatocarcinogenesis, HIFs act as master regulators to dampen the innate immunity in the liver to allow cancer cells to bypass immune surveillance. There are many exciting research questions yet to be addressed. In the future, studies should focus on the functions of hypoxia/HIFs in the complement system and the antigen presentation abilities of different innate immune cells in liver cancer. Innate immunity is tightly linked with adaptive immunity, and together they affect the response to immune therapies. Due to space limitation, we have only covered the roles of hypoxia/HIFs in innate immune cells in liver cancer. HIFs also orchestrate the adaptive immune system by transcriptionally activating genes important for Treg enrichment and $\mathrm{T}$ cell exhaustion. In the future, researchers should take advantage of new technol- 
ogy platforms such as single-cell RNA sequencing, mass cytometry (CyTOF), and fluorescent multiplex immunohistochemistry to obtain a complete understanding of the involvement of HIFs in innate and adaptive immune system interactions in different stages of hepatocarcinogenesis. As immune checkpoint blockade has emerged as a next-generation therapy for liver cancer, and blockade of the HIF signaling pathway is expected to subvert the immunosuppressive microenvironment and restore $\mathrm{T}$ cell infiltration, more translational efforts should be put forth to evaluate the effects of different HIF inhibitors in combination with various immune checkpoint inhibitors for liver cancer treatment.

\section{Acknowledgments}

This work was supported by the Research Grant Council-ThemeBased Research Fund (T12-704/16-R), State Key Laboratory of Liver Research, Croucher Innovation Award, and University of Hong Kong Outstanding Young Researcher Award.

Address correspondence to: Carmen Chak-Lui Wong, Department of Pathology, State Key Laboratory of Liver Research, University of Hong Kong, T810, Block T, Queen Mary Hospital, Pokfulam, Hong Kong, China. Phone: 852.2255.2689; Email: carmencl@pathology.hku.hk.
1. Colgan SP, Furuta GT, Taylor CT. Hypoxia and innate immunity: keeping up with the HIFsters. Annu Rev Immunol. 2020;38:341-363.

2. Taylor CT, Colgan SP. Regulation of immunity and inflammation by hypoxia in immunological niches. Nat Rev Immunol. 2017;17(12):774-785.

3. Bray F, Ferlay J, Soerjomataram I, Siegel RL, Torre LA, Jemal A. Global cancer statistics 2018: GLOBOCAN estimates of incidence and mortality worldwide for 36 cancers in 185 countries. CA Cancer JClin. 2018;68(6):394-424.

4. Sia D, Villanueva A, Friedman SL, Llovet JM. Liver cancer cell of origin, molecular class, and effects on patient prognosis. Gastroenterology. 2017;152(4):745-761.

5. Abou-Alfa GK, et al. Cabozantinib in patients with advanced and progressing hepatocellular carcinoma. N Engl J Med. 2018;379(1):54-63.

6. Ikeda M, et al. Safety and pharmacokinetics of lenvatinib in patients with advanced hepatocellular carcinoma. Clin Cancer Res. 2016;22(6):1385-1394.

7. Llovet JM, et al. Sorafenib in advanced hepatocellular carcinoma. N Engl JMed. 2008;359(4):378-390.

8. El-Khoueiry AB, et al. Nivolumab in patients with advanced hepatocellular carcinoma (CheckMate 040): an open-label, non-comparative, phase $1 / 2$ dose escalation and expansion trial. Lancet. 2017;389(10088):2492-2502.

9. Gao B. Basic liver immunology. Cell Mol Immunol. 2016;13(3):265-266.

10. Heymann F, Tacke F. Immunology in the liverfrom homeostasis to disease. Nat Rev Gastroenterol Hepatol. 2016;13(2):88-110.

11. Ringelhan M, Pfister D, O'Connor T, Pikarsky E, Heikenwalder M. The immunology of hepatocellular carcinoma. Nat Immunol. 2018;19(3):222-232.

12. Bronte $\mathrm{V}$, et al. Recommendations for myeloid-derived suppressor cell nomenclature and characterization standards. Nat Commun. 2016;7:12150

13. Gabrilovich DI. Myeloid-derived suppressor cells. Cancer Immunol Res. 2017;5(1):3-8.

14. Qian BZ, Pollard JW. Macrophage diversity enhances tumor progression and metastasis. Cell. 2010;141(1):39-51.

15. Mak TW, Saunders ME, Jett B. Primer to the Immune Response. Elsevier; 2014.

16. Liang W, Ferrara N. The complex role of neutrophils in tumor angiogenesis and metastasis. Cancer Immunol Res. 2016;4(2):83-91.

17. Terrén I, Orrantia A, Vitallé J, Zenarruzabeitia O, Borrego F. NK cell metabolism and tumor microenvironment. Front Immunol. 2019;10:2278.
18. Jungermann K, Kietzmann T. Oxygen: modulator of metabolic zonation and disease of the liver. Hepatology. 2000;31(2):255-260.

19. Vaupel P, Höckel M, Mayer A. Detection and characterization of tumor hypoxia using $\mathrm{pO} 2$ histography. Antioxid Redox Signal. 2007;9(8):1221-1235.

20. Roth KJ, Copple BL. Role of hypoxia-inducible factors in the development of liver fibrosis. Cell Mol Gastroenterol Hepatol. 2015;1(6):589-597.

21. Sun X, et al. Antisense hypoxia-inducible factor-1alpha augments transcatheter arterial embolization in the treatment of hepatocellular carcinomas in rats. Hum Gene Ther. 2009;20(4):314-324.

22. Méndez-Blanco C, Fondevila F, García-Palomo A, González-Gallego J, Mauriz JL. Sorafenib resistance in hepatocarcinoma: role of hypoxia-inducible factors. Exp Mol Med. 2018;50(10):1-9.

23. Jiang BH, Rue E, Wang GL, Roe R, Semenza GL. Dimerization, DNA binding, and transactivation properties of hypoxia-inducible factor 1. J Biol Chem. 1996;271(30):17771-17778.

24. Wang GL, Semenza GL. Purification and characterization of hypoxia-inducible factor 1. J Biol Chem. 1995;270(3):1230-1237.

25. Epstein AC, et al. C. elegans EGL-9 and mammalian homologs define a family of dioxygenases that regulate HIF by prolyl hydroxylation. Cell. 2001;107(1):43-54

26. Kaelin WG, Ratcliffe PJ. Oxygen sensing by metazoans: the central role of the HIF hydroxylase pathway. Mol Cell. 2008;30(4):393-402.

27. Wang GL, Jiang BH, Rue EA, Semenza GL. Hypoxia-inducible factor 1 is a basic-helixloop-helix-PAS heterodimer regulated by cellular O2 tension. Proc Natl Acad Sci U S A. 1995;92(12):5510-5514.

28. Copple BL, Bai S, Moon JO. Hypoxia-inducible factor-dependent production of profibrotic mediators by hypoxic Kupffer cells. Hepatol Res. 2010;40(5):530-539.

29. Copple BL, Bustamante JJ, Welch TP, Kim ND, Moon JO. Hypoxia-inducible factor-dependent production of profibrotic mediators by hypoxic hepatocytes. Liver Int. 2009;29(7):1010-1021.

30. Copple BL, Kaska S, Wentling C. Hypoxia-inducible factor activation in myeloid cells contributes to the development of liver fibrosis in cholestatic mice. J Pharmacol Exp Ther. 2012;341(2):307-316

31. Friedman SL, Arthur MJ. Activation of cultured rat hepatic lipocytes by Kupffer cell conditioned medium. Direct enhancement of matrix synthesis and stimulation of cell proliferation via induc- tion of platelet-derived growth factor receptors. JClin Invest. 1989;84(6):1780-1785.

32. Tsuchida T, Friedman SL. Mechanisms of hepatic stellate cell activation. Nat Rev Gastroenterol Hepatol. 2017;14(7):397-411.

33. Ankoma-Sey V, Wang Y, Dai Z. Hypoxic stimulation of vascular endothelial growth factor expression in activated rat hepatic stellate cells. Hepatology. 2000;31(1):141-148.

34. Kantari-Mimoun C, et al. Resolution of liver fibrosis requires myeloid cell-driven sinusoidal angiogenesis. Hepatology. 2015;61(6):2042-2055.

35 . Yang $\mathrm{L}$, et al. Vascular endothelial growth factor promotes fibrosis resolution and repair in mice. Gastroenterology. 2014;146(5):1339-50.e1.

36. Qu A, et al. Hypoxia-inducible transcription factor $2 \alpha$ promotes steatohepatitis through augmenting lipid accumulation, inflammation, and fibrosis. Hepatology. 2011;54(2):472-483.

37. Mochizuki A, et al. Hepatic stellate cells orchestrate clearance of necrotic cells in a hypoxia-inducible factor- $1 \alpha$-dependent manner by modulating macrophage phenotype in mice. Jimmunol. 2014;192(8):3847-3857.

38. Bangoura G, Yang LY, Huang GW, Wang W. Expression of HIF-2alpha/EPAS1 in hepatocellular carcinoma. World J Gastroenterol. 2004;10(4):525-530.

39. Huang GW, Yang LY, Lu WQ. Expression of hypoxia-inducible factor 1alpha and vascular endothelial growth factor in hepatocellular carcinoma: impact on neovascularization and survival. World J Gastroenterol. 2005;11(11):1705-1708

40. Wong CC, Kai AK, Ng IO. The impact of hypoxia in hepatocellular carcinoma metastasis. Front Med. 2014;8(1):33-41.

41. Bangoura G, Liu ZS, Qian Q, Jiang CQ, Yang GF, Jing S. Prognostic significance of HIF-2alpha/ EPAS1 expression in hepatocellular carcinoma. World J Gastroenterol. 2007;13(23):3176-3182.

42. Xiang ZL, Zeng ZC, Fan J, Tang ZY, Zeng HY, Gao DM. Gene expression profiling of fixed tissues identified hypoxia-inducible factor-1 $\alpha$, VEGF, and matrix metalloproteinase- 2 as biomarkers of lymph node metastasis in hepatocellular carcinoma. Clin Cancer Res. 2011;17(16):5463-5472.

43. van Malenstein H, et al. A seven-gene set associated with chronic hypoxia of prognostic importance in hepatocellular carcinoma. Clin Cancer Res. 2010;16(16):4278-4288.

44. Ma L, et al. Tumor cell biodiversity drives microenvironmental reprogramming in liver cancer. Cancer Cell. 2019;36(4):418-430.e6. 
45. Zhang Q, et al. Landscape and dynamics of single immune cells in hepatocellular carcinoma. Cell. 2019;179(4):829-845.e20.

46. Cramer T, et al. HIF-1alpha is essential for myeloid cell-mediated inflammation. Cell. 2003;112(5):645-657.

47. Imtiyaz HZ, et al. Hypoxia-inducible factor 2alpha regulates macrophage function in mouse models of acute and tumor inflammation. J Clin Invest. 2010;120(8):2699-2714.

48. Wu Q, et al. Blocking triggering receptor expressed on myeloid cells-1-positive tumor-associated macrophages induced by hypoxia reverses immunosuppression and anti-programmed cell death ligand 1 resistance in liver cancer. Hepatology. 2019;70(1):198-214.

49. Rius J, et al. NF-kappaB links innate immunity to the hypoxic response through transcriptional regulation of HIF-1alpha. Nature. 2008;453(7196):807-811.

50. Koh MY, Darnay BG, Powis G. Hypoxia-associated factor, a novel E3-ubiquitin ligase, binds and ubiquitinates hypoxia-inducible factor 1alpha, leading to its oxygen-independent degradation. Mol Cell Biol. 2008;28(23):7081-7095.

51. Koh MY, et al. A new HIF-1 $\alpha /$ RANTES-driven pathway to hepatocellular carcinoma mediated by germline haploinsufficiency of SART1/HAF in mice. Hepatology. 2016;63(5):1576-1591.

52. White JR, et al. Genetic amplification of the transcriptional response to hypoxia as a novel means of identifying regulators of angiogenesis. Genomics. 2004;83(1):1-8.

53. Eubank TD, Roda JM, Liu H, O'Neil T, Marsh CB. Opposing roles for HIF- $1 \alpha$ and HIF- $2 \alpha$ in the regulation of angiogenesis by mononuclear phagocytes. Blood. 2011;117(1):323-332.

54. Du R, et al. HIF1alpha induces the recruitment of bone marrow-derived vascular modulatory cells to regulate tumor angiogenesis and invasion. Cancer Cell. 2008;13(3):206-220.

55. De Palma M, et al. Tie2 identifies a hematopoietic lineage of proangiogenic monocytes required for tumor vessel formation and a mesenchymal population of pericyte progenitors. Cancer Cell. 2005;8(3):211-226.

56. Matsubara T, et al. TIE2-expressing monocytes as a diagnostic marker for hepatocellular carcinoma correlates with angiogenesis. Hepatology. 2013;57(4):1416-1425.

57. Peng SH, et al. Significance and relationship between infiltrating inflammatory cell and tumor angiogenesis in hepatocellular carcinoma tissues. World J Gastroenterol. 2005;11(41):6521-6524.

58. Corzo CA, et al. HIF-1 $\alpha$ regulates function and differentiation of myeloid-derived suppressor cells in the tumor microenvironment. JExp Med. 2010;207(11):2439-2453.

59. Noman MZ, et al. Tumor-promoting effects of myeloid-derived suppressor cells are potentiated by hypoxia-induced expression of miR-210. Cancer Res. 2015;75(18):3771-3787.

60. Noman MZ, et al. PD-L1 is a novel direct target of HIF-1 $\alpha$, and its blockade under hypoxia enhanced MDSC-mediated T cell activation. JExp Med. 2014;211(5):781-790.

61. Freeman GJ, et al. Engagement of the PD-1 immunoinhibitory receptor by a novel B7 family member leads to negative regulation of lymphocyte activation. JExp Med. 2000;192(7):1027-1034.

62. Kuang DM, et al. Activated monocytes in peritumoral stroma of hepatocellular carcinoma foster immune privilege and disease progression through PD-L1.JExp Med. 2009;206(6):1327-1337.

63. Walmsley SR, et al. Hypoxia-induced neutrophil survival is mediated by HIF-1alpha-dependent NF-kappaB activity.JExp Med.2005;201(1):105-115.

64. Dumitru CA, Lang S, Brandau S. Modulation of neutrophil granulocytes in the tumor microenvironment: mechanisms and consequences for tumor progression. Semin Cancer Biol.2013;23(3):141-148.

65. Phan VT, et al. Oncogenic RAS pathway activation promotes resistance to anti-VEGF therapy through G-CSF-induced neutrophil recruitment. Proc Natl Acad Sci US A. 2013;110(15):6079-6084.

66. Fridlender ZG, et al. Polarization of tumor-associated neutrophil phenotype by TGF-beta: "N1" versus "N2" TAN. Cancer Cell. 2009;16(3):183-194.

67. Wilson CL, et al. NFkB1 is a suppressor of neutrophil-driven hepatocellular carcinoma. Nat Commun. 2015;6:6818.

68. Jiang Y, Dai A, Li Q, Hu R. Hypoxia induces transforming growth factor-beta1 gene expression in the pulmonary artery of rats via hypoxia-inducible factor-1alpha. Acta Biochim Biophys Sin (Shanghai). 2007;39(1):73-80.

69. McMahon S, Charbonneau M, Grandmont S, Richard DE, Dubois CM. Transforming growth factor beta1 induces hypoxia-inducible factor-1 stabilization through selective inhibition of PHD2 expression. J Biol Chem. 2006;281(34):24171-24181.

70. Wong CC, et al. Lysyl oxidase-like 2 is critical to tumor microenvironment and metastatic niche formation in hepatocellular carcinoma. Hepatology. 2014;60(5):1645-1658.

71. Zhou SL, et al. Tumor-associated neutrophils recruit macrophages and T-regulatory cells to promote progression of hepatocellular carcinoma and resistance to sorafenib. Gastroenterology. 2016;150(7):1646-1658.e17.

72. Balsamo M, et al. Hypoxia downregulates the expression of activating receptors involved in NK-cell-mediated target cell killing without affecting ADCC. Eur J Immunol. 2013;43(10):2756-2764.

73. Kamimura H, et al. Reduced NKG2D ligand expression in hepatocellular carcinoma correlates with early recurrence. J Hepatol. 2012;56(2):381-388.

74. Yaghi L, et al. Hypoxia inducible factor-1 mediates the expression of the immune checkpoint HLA-G in glioma cells through hypoxia response element located in exon 2. Oncotarget. 2016;7(39):63690-63707.

75. Amiot L, Vu N, Samson M. Biology of the immunomodulatory molecule HLA-G in human liver diseases. J Hepatol. 2015;62(6):1430-1437.

76. Lin A, et al. Aberrant human leucocyte antigen-G expression and its clinical relevance in hepatocellular carcinoma. JCell MolMed.2010;14(8):2162-2171.

77. Tosello-Trampont AC, Krueger P, Narayanan S, Landes SG, Leitinger N, Hahn YS. NKp46(+) natural killer cells attenuate metabolism-induced hepatic fibrosis by regulating macrophage activation in mice. Hepatology. 2016;63(3):799-812.

78. Hoechst B, et al. Myeloid derived suppressor cells inhibit natural killer cells in patients with hepatocellular carcinoma via the NKp30 receptor. Hepatology. 2009;50(3):799-807.

79. Ye LY, et al. Hypoxia-induced epithelial-to-mesenchymal transition in hepatocellular carcinoma induces an immunosuppressive tumor microenvironment to promote metastasis. Cancer Res. 2016;76(4):818-830.

80. Adams JL, Smothers J, Srinivasan R, Hoos A. Big opportunities for small molecules in immuno-oncology. Nat Rev Drug Discov. 2015;14(9):603-622.

81. Mellor AL, Munn DH. IDO expression by dendritic cells: tolerance and tryptophan catabolism. Nat Rev Immunol. 2004;4(10):762-774.

82. Casazza A, et al. Impeding macrophage entry into hypoxic tumor areas by Sema3A/Nrp1 signaling blockade inhibits angiogenesis and restores antitumor immunity. Cancer Cell.2013;24(6):695-709.

83. Hu ZQ, et al. Overexpression of semaphorin 3A promotes tumor progression and predicts poor prognosis in hepatocellular carcinoma after curative resection. Oncotarget. 2016;7(32):51733-51746.

84. Li X, et al. Novel role of semaphorin $3 \mathrm{~A}$ in the growth and progression of hepatocellular carcinoma. Oncol Rep. 2017;37(6):3313-3320.

85. Barleon B, Sozzani S, Zhou D, Weich HA, Mantovani A, Marmé D. Migration of human monocytes in response to vascular endothelial growth factor (VEGF) is mediated via the VEGF receptor flt-1. Blood. 1996;87(8):3336-3343.

86. Sawano A, et al. Flt-1, vascular endothelial growth factor receptor 1 , is a novel cell surface marker for the lineage of monocyte-macrophages in humans. Blood. 2001;97(3):785-791.

87. Poon RT, Fan ST, Wong J. Clinical implications of circulating angiogenic factors in cancer patients. JClin Oncol. 2001;19(4):1207-1225.

88. von Marschall Z, Cramer T, Höcker M, Finkenzeller G, Wiedenmann B, Rosewicz S. Dual mechanism of vascular endothelial growth factor upregulation by hypoxia in human hepatocellular carcinoma. Gut. 2001;48(1):87-96.

89. Chiu DK, et al. Hypoxia induces myeloid-derived suppressor cell recruitment to hepatocellular carcinoma through chemokine (C-C motif) ligand 26. Hepatology. 2016;64(3):797-813.

90. Shinkai A, et al. A novel human CC chemokine, eotaxin-3, which is expressed in IL-4-stimulated vascular endothelial cells, exhibits potent activity toward eosinophils. JImmunol. 1999;163(3):1602-1610.

91. Erler JT, et al. Hypoxia-induced lysyl oxidase is a critical mediator of bone marrow cell recruitment to form the premetastatic niche. Cancer Cell. 2009;15(1):35-44.

92. Wong CC, et al. Hypoxia-inducible factor 1 is a master regulator of breast cancer metastatic niche formation. Proc Natl Acad Sci U S A. 2011;108(39):16369-16374.

93. Pallett LJ, et al. Metabolic regulation of hepatitis B immunopathology by myeloid-derived suppressor cells. Nat Med. 2015;21(6):591-600.

94. Thomas S, et al. CD24 is an effector of HIF-1driven primary tumor growth and metastasis. Cancer Res. 2012;72(21):5600-5612.

95. Zhang $\mathrm{H}$, et al. HIF-1 regulates CD47 expression in breast cancer cells to promote evasion of phagocytosis and maintenance of 
cancer stem cells. Proc Natl Acad Sci U S A. 2015;112(45):E6215-E6223.

96. Chao MP, Majeti R, Weissman IL. Programmed cell removal: a new obstacle in the road to developing cancer. Nat Rev Cancer. 2011;12(1):58-67.

97. Barkal AA, et al. CD24 signalling through macrophage Siglec-10 is a target for cancer immunotherapy. Nature. 2019;572(7769):392-396.

98. Lee TK, Castilho A, Cheung VC, Tang KH, Ma S, Ng IO. CD24(+) liver tumor-initiating cells drive self-renewal and tumor initiation through STAT3-mediated NANOG regulation. Cell Stem Cell. 2011;9(1):50-63.

99. Lee TK, et al. Blockade of CD47-mediated cathepsin S/protease-activated receptor 2 signaling provides a therapeutic target for hepatocellular carcinoma. Hepatology. 2014;60(1):179-191.

100.Cui CP, et al. SENP1 promotes hypoxia-induced cancer stemness by HIF-1 $\alpha$ deSUMOylation and SENP1/HIF-1 $\alpha$ positive feedback loop. Gut. 2017;66(12):2149-2159.

101. Yamashita T, Wang XW. Cancer stem cells in the development of liver cancer. J Clin Invest. 2013;123(5):1911-1918.

102. Antonioli L, Blandizzi C, Pacher P, Haskó G. Immunity, inflammation and cancer: a leading role for adenosine. Nat Rev Cancer. 2013;13(12):842-857.

103. Sitkovsky M, Lukashev D. Regulation of immune cells by local-tissue oxygen tension: HIF1 alpha and adenosine receptors. Nat Rev Immunol. 2005;5(9):712-721.

104.Van Belle H, Goossens F, Wynants J. Formation and release of purine catabolites during hypoperfusion, anoxia, and ischemia. Am J Physiol. 1987;252(5 Pt 2):H886-H893.

105. Allard B, Pommey S, Smyth MJ, Stagg J. Targeting CD73 enhances the antitumor activity of antiPD-1 and anti-CTLA-4 mAbs. Clin Cancer Res. 2013;19(20):5626-5635.

106. Beavis PA, et al. Blockade of A2A receptors potently suppresses the metastasis of CD73+ tumors. Proc Natl Acad Sci U S A. 2013;110(36):14711-14716.

107. Csóka B, et al. Adenosine promotes alternative macrophage activation via $\mathrm{A} 2 \mathrm{~A}$ and $\mathrm{A} 2 \mathrm{~B}$ receptors. FASEB J. 2012;26(1):376-386.

108.Lokshin A, Raskovalova T, Huang X, Zacharia LC, Jackson EK, Gorelik E. Adenosine-mediated inhibition of the cytotoxic activity and cytokine production by activated natural killer cells. Cancer Res. 2006;66(15):7758-7765.

109. Ohta A, Kini R, Ohta A, Subramanian M, Madasu M, Sitkovsky M. The development and immunosuppressive functions of CD4(+) CD25(+) FoxP3(+) regulatory $\mathrm{T}$ cells are under influence of the adenosine-A2A adenosine receptor pathway. Front Immunol. 2012;3:190.

110. Raskovalova T, Huang X, Sitkovsky M, Zacharia LC Jackson EK, Gorelik E. Gs protein-coupled adenosine receptor signaling and lytic function of activated NK cells. JImmunol. 2005;175(7):4383-4391.

111. Raskovalova T, Lokshin A, Huang X, Jackson EK, Gorelik E. Adenosine-mediated inhibition of cytotoxic activity and cytokine production by IL-2/NKp46-activated NK cells: involvement of protein kinase A isozyme I (PKA I). Immunol Res.
2006;36(1-3):91-99.

112. Sevigny CP, et al. Activation of adenosine 2 A receptors attenuates allograft rejection and alloantigen recognition. J Immunol. 2007;178(7):4240-4249.

113. Novitskiy SV, et al. Adenosine receptors in regulation of dendritic cell differentiation and function. Blood. 2008;112(5):1822-1831.

114. Ryzhov S, et al. Adenosinergic regulation of the expansion and immunosuppressive activity of CD11b+Gr1+ cells. J Immunol. 2011;187(11):6120-6129.

115. Yang M, et al. HIF-dependent induction of adenosine receptor $\mathrm{A} 2 \mathrm{~b}$ skews human dendritic cells to a Th2-stimulating phenotype under hypoxia. Immunol Cell Biol. 2010;88(2):165-171.

116. Li XY, et al. Targeting CD39 in cancer reveals an extracellular ATP- and inflammasome-driven tumor immunity. Cancer Discov. 2019;9(12):1754-1773.

117. Stagg J, et al. Anti-CD73 antibody therapy inhibits breast tumor growth and metastasis. Proc Natl Acad Sci U S A. 2010;107(4):1547-1552.

118. Chiu DK, et al. Hypoxia inducible factor HIF-1 promotes myeloid-derived suppressor cells accumulation through ENTPD2/CD39L1 in hepatocellular carcinoma. Nat Commun. 2017;8(1):517.

119. Samanta D, et al. Chemotherapy induces enrichment of $\mathrm{CD} 47^{+} / \mathrm{CD} 3^{+} / \mathrm{PDL}^{+}$immune evasive triple-negative breast cancer cells. Proc Natl Acad Sci U S A. 2018;115(6):E1239-E1248.

120. Synnestvedt K, et al. Ecto-5'-nucleotidase (CD73) regulation by hypoxia-inducible factor-1 mediates permeability changes in intestinal epithelia. J Clin Invest. 2002;110(7):993-1002.

121. Ma DF, et al. Hypoxia-inducible adenosine A2B receptor modulates proliferation of colon carcinoma cells. Hum Pathol. 2010;41(11):1550-1557.

122. Sun $\mathrm{X}$, et al. Disordered purinergic signaling and abnormal cellular metabolism are associated with development of liver cancer in Cd39/ENTPD1 null mice. Hepatology. 2013;57(1):205-216.

123. Feldbrügge L, et al. Distinct roles of ecto-nucleoside triphosphate diphosphohydrolase-2 (NTPDase2) in liver regeneration and fibrosis. Purinergic Signal. 2018;14(1):37-46.

124. Rothweiler S, et al. Selective deletion of ENTPD1/CD39 in macrophages exacerbates biliary fibrosis in a mouse model of sclerosing cholangitis. Purinergic Signal. 2019;15(3):375-385.

125. Savio LEB, et al. CD39 limits P2X7 receptor inflammatory signaling and attenuates sepsis-induced liver injury. J Hepatol. 2017;67(4):716-726.

126. Tak E, et al. Protective role of hypoxia-inducible factor- $1 \alpha$-dependent CD39 and CD73 in fulminant acute liver failure. Toxicol Appl Pharmacol. 2017;314:72-81.

127. Zhang J, et al. Hypoxia-inducible factor- $1 \alpha /$ interleukin- $1 \beta$ signaling enhances hepatoma epithelial-mesenchymal transition through macrophages in a hypoxic-inflammatory microenvironment. Hepatology. 2018;67(5):1872-1889.

128. Chen X, Calvisi DF. Hydrodynamic transfection for generation of novel mouse models for liver cancer research. Am J Pathol. 2014;184(4):912-923.

129. Xue W, et al. CRISPR-mediated direct mutation of cancer genes in the mouse liver. Nature.
2014;514(7522):380-384.

130. Zhang H, et al. Digoxin and other cardiac glycosides inhibit HIF-1alpha synthesis and block tumor growth. Proc Natl Acad Sci US A. 2008;105(50):19579-19586.

131. Samanta D, Gilkes DM, Chaturvedi P, Xiang L, Semenza GL. Hypoxia-inducible factors are required for chemotherapy resistance of breast cancer stem cells. Proc Natl Acad Sci U S A. 2014;111(50):E5429-E5438.

132. Schito L, et al. Hypoxia-inducible factor 1-dependent expression of platelet-derived growth factor B promotes lymphatic metastasis of hypoxic breast cancer cells. Proc Natl Acad Sci U S A. 2012;109(40):E2707-E2716.

133. Wong CC, et al. Inhibitors of hypoxia-inducible factor 1 block breast cancer metastatic niche formation and lung metastasis. J Mol Med. 2012;90(7):803-815.

134.Zhang $\mathrm{H}$, et al. HIF-1-dependent expression of angiopoietin-like 4 and L1CAM mediates vascular metastasis of hypoxic breast cancer cells to the lungs. Oncogene. 2012;31(14):1757-1770.

135. Greenberger LM, et al. A RNA antagonist of hypoxia-inducible factor-1alpha, EZN-2968, inhibits tumor cell growth. Mol Cancer Ther. 2008;7(11):3598-3608.

136. Wu J, et al. Evaluation of a locked nucleic acid form of antisense oligo targeting HIF- $1 \alpha$ in advanced hepatocellular carcinoma. World J Clin Oncol. 2019;10(3):149-160.

137. Liang Y, et al. Hypoxia-mediated sorafenib resistance can be overcome by EF24 through Von Hippel-Lindau tumor suppressor-dependent HIF-1 $\alpha$ inhibition in hepatocellular carcinoma. Hepatology. 2013;57(5):1847-1857.

138. Thomas SL, et al. EF24, a novel curcumin analog, disrupts the microtubule cytoskeleton and inhibits HIF-1. Cell Cycle. 2008;7(15):2409-2417.

139. Xu J, et al. Increasing AR by HIF-2 $\alpha$ inhibitor (PT2385) overcomes the side-effects of sorafenib by suppressing hepatocellular carcinoma invasion via alteration of pSTAT3, pAKT and pERK signals. Cell Death Dis. 2017;8(10):e3095.

140.Zhang W, et al. Depletion of tumor-associated macrophages enhances the effect of sorafenib in metastatic liver cancer models by antimetastatic and antiangiogenic effects. Clin Cancer Res. 2010;16(13):3420-3430.

141. Finn RS, et al. Atezolizumab plus bevacizumab in unresectable hepatocellular carcinoma. $N$ Engl J Med.2020;382(20):1894-1905.

142. Shigeta K, et al. Dual programmed death receptor-1 and vascular endothelial growth factor receptor-2 blockade promotes vascular normalization and enhances antitumor immune responses in hepatocellular carcinoma. Hepatology. 2020;71(4):1247-1261.

143. Sitohy B, Nagy JA, Dvorak HF. Anti-VEGF/ VEGFR therapy for cancer: reassessing the target. Cancer Res. 2012;72(8):1909-1914.

144.Ueda S, Saeki T, Osaki A, Yamane T, Kuji I. Bevacizumab induces acute hypoxia and cancer progression in patients with refractory breast cancer: multimodal functional imaging and multiplex cytokine analysis. Clin Cancer Res. 2017;23(19):5769-5778. 